\title{
Histidine and arginine are associated with inflammation and oxidative stress in obese women
}

\author{
Yu-Cun Niu ${ }^{1} \dagger$, Ren-Nan Feng ${ }^{1} \dagger$, Yan $\mathrm{Hou}^{2}$, Kang $\mathrm{Li}^{2}$, Zhen Kang ${ }^{3}$, Jian $\mathrm{Wang}^{3}$, Chang-Hao Sun ${ }^{1 *}$ \\ and Ying $\mathrm{Li}^{1 *}$ \\ ${ }^{1}$ Department of Nutrition and Food Hygiene, School of Public Health, Harbin Medical University, Harbin, People's Republic \\ of China \\ ${ }^{2}$ Department of Biostatistics, School of Public Health, Harbin Medical University, Harbin, People's Republic of China \\ ${ }^{3}$ Harbin Center for Disease Control and Prevention, Harbin, People's Republic of China
}

(Submitted 17 January 2011 - Final revision received 25 August 2011 - Accepted 25 August 2011 - First published online 14 October 2011)

\section{Abstract}

The aims of the present study were to examine the serum amino acid profiles in obese and non-obese women and investigate the relationships between the serum amino acids and inflammation and oxidative stress in a human case-control study. Serum amino acids, inflammatory biomarkers (C-reactive protein and IL-6) and oxidative biomarkers (superoxide dismutase, malondialdehyde and glutathione peroxidase) were measured and compared in 235 obese women and 217 non-obese controls. The relationships between serum amino acids and inflammatory and oxidative biomarkers were examined using multiple linear regression. Among the amino acids determined, serum histidine, arginine, threonine, glycine, lysine and serine were found to be significantly lower in obese women as compared to non-obese controls $(P<0 \cdot 001)$. The difference was the greatest for histidine $(P<0 \cdot 001)$. In obese women, both histidine and arginine were negatively associated with inflammation and oxidative stress. In non-obese controls, histidine was negatively associated with oxidative stress. The findings in this study indicate that the metabolism of amino acids is abnormal in obese women in whom histidine and arginine have close relationships with inflammation and oxidative stress.

\section{Key words: Amino acids: Inflammation: Oxidative stress: Obesity}

Obesity is a major risk factor for a number of chronic diseases, including type 2 diabetes, hypertension and $\mathrm{CVD}^{(1)}$. Increased levels of inflammatory and oxidative biomarkers are the main features of obesity, which is the pathological and physiological feature of many conditions ${ }^{(2)}$. Obesity could lead to metabolic disorders of blood glucose and lipids ${ }^{(3,4)}$. And numerous studies $^{(5-8)}$ have confirmed that the metabolic disorders of blood glucose and lipids are closely related to inflammation and oxidative stress. However, it is still unclear if amino acid metabolism is altered and if there are links between serum amino acids and inflammation and oxidative stress in obese subjects.

As carbohydrate, protein and fat are the main macronutrients, whose metabolisms are closely related with each other, we hypothesised that imbalanced amino acid metabolism might also exist in obese subjects. The aims of this study were to compare the serum amino acid profiles between obese and non-obese women, and evaluate the associations between amino acid metabolism and inflammation and oxidative stress in a human case-control study.

\section{Methods}

A total of 235 middle-aged obese women and 217 non-obese controls were recruited into the present study based on the criteria: (1) BMI of $18-24 \mathrm{~kg} / \mathrm{m}^{2}$ (non-obese control group); $\mathrm{BMI} \geq 28 \mathrm{~kg} / \mathrm{m}^{2}$ (obese group) ${ }^{(9)}$; (2) fasting blood glucose $<6.1 \mathrm{mmol} / 1$ and 2 -h postload blood glucose concentration $<7.8 \mathrm{mmol} / \mathrm{l}$; (3) BMI fluctuation $<0.5 \mathrm{~kg} / \mathrm{m}^{2}$ in the past 6 months; (4) no disease that could influence the metabolism of nutrients, such as kidney, liver and gastrointestinal diseases; (5) no history of endocrine metabolic disorders, acute infection, injuries or chronic inflammatory diseases; (6) not being pregnant. This study was conducted according to the guidelines laid down in the Declaration of Helsinki and all procedures involving human subjects were approved by

Abbreviations: CRP, C-reactive protein; MDA, malondialdehyde; SOD, superoxide dismutase.

*Corresponding authors: Chang-Hao Sun, fax +86 451 87502885, email sun2002changhao@yahoo.com; Ying Li, fax +86 451 87502885, email liying2002helen@gmail.com

† Authors contributed equally to this work. 
the Human Research Ethics Committee of the Harbin Medical University. Written informed consent was obtained from all subjects.

Information on age, weight, height, alcohol use, cigarette smoking, menstrual status, and physical activity at work and at leisure was obtained from questionnaires. Dietary intakes were measured with a validated semi-quantitative FFQ based on frequency ${ }^{(10)}$. Energy and nutrient intakes were calculated with the Food Nutrition Calculator (V1.60; Chinese CDC, Beijing, China).

Using a standard protocol, trained examiners measured body weight $( \pm 0 \cdot 1 \mathrm{~kg})$, height $( \pm 0 \cdot 1 \mathrm{~cm})$ and the blood pressure of all participants. BMI was calculated as body weight (kg) divided by the square of height $(\mathrm{m})$. Blood pressure was measured with a standard mercury sphygmomanometer on the right arm after resting for at least $10 \mathrm{~min}$. The mean of the three closest readings was used in the analysis.

Subjects were instructed to fast for $12 \mathrm{~h}$ and blood samples were collected in the morning. After the fasting blood sample collection, each subject was given an oral glucose tolerance test and blood samples were collected in $2 \mathrm{~h}$ after the $75 \mathrm{~g}$ glucose was taken. Antecubital venous blood was collected to obtain serum and plasma, and then stored at $-80^{\circ} \mathrm{C}$.

Samples of serum $(100 \mu \mathrm{l})$ were spiked with acetonitrile $(100 \mu \mathrm{l})$, vortex-mixed for $30 \mathrm{~s}$, immediately centrifuged at $11000 \mathrm{~g}$ for $10 \mathrm{~min}$ at $4^{\circ} \mathrm{C}$ and the supernatant was recovered. An Alliance 2695 separation module and a 2487 UV detector from Waters were used for the HPLC analysis (CV\%, 4.2$5 \cdot 3 \%)$. Derivatisation and gradient elution of serum amino acids were followed according to the manufacturer's instructions (Waters Corporation, Milford, MA, USA).

Fasting blood glucose, 2-h postload blood glucose, total cholesterol, HDL-cholesterol and TAG were determined using commercial kits (Biosino Biotechnology, Beijing, China), standard enzymatic colorimetric techniques and an auto-analyser (MOL-300, Shanghai, China).

C-reactive protein (CRP) and IL-6 were assayed using ELISA with commercial kits (CRP, Biocheck, Inc., Foster City, CA, USA; IL-6, R\&D Systems Europe, Abingdon, UK) according to the manufacturers' instructions. Superoxide dismutase (SOD), malondialdehyde (MDA) and glutathione peroxidase were measured with commercial kits using enzymatic methods (Jiancheng Technology, Nanjing, China).

\section{Statistical analysis}

Data were presented as means and standard deviations. Comparisons between obese and non-obese women in the population study were made by using Student's $t$ test for continuous variables and $\chi^{2}$ test for categorical variables. ANCOVA was used to compare serum amino acid levels between obese women and non-obese controls, adjusting for potential covariates (age, smoking, alcohol use, menopause, and physical activity at work and at leisure). Multiple linear regression (adjusted for the aforementioned variables) between serum amino acids and inflammatory and oxidative biomarkers was also calculated in overall, non-obese controls and obese women, respectively. Statistical analyses were carried out with SAS software (version 9.1; SAS Institute, Inc., Cary, NC, USA).

\section{Results}

\section{Clinical parameters}

As shown in Table 1, BMI, systolic blood pressure, diastolic blood pressure, 2-h postload blood glucose concentration, total cholesterol, TAG, fat and energy intakes (data not shown), CRP, IL-6 and MDA were significantly higher, while HDL-cholesterol, SOD and glutathione peroxidase of obese women were significantly lower than that of non-obese controls. There were no significant differences in the other variables (smoking, alcohol drinking, menopause and physical activity; data not shown).

\section{Amino acid profile}

The serum concentrations of amino acids in obese women and non-obese controls are presented in Table 1. Accounting

Table 1. Clinical parameters and amino acid profiles of obese women and non-obese controls

(Mean values and standard deviations)

\begin{tabular}{|c|c|c|c|c|}
\hline & \multicolumn{2}{|c|}{$\begin{array}{l}\text { Obese women } \\
\quad(n \text { 235) }\end{array}$} & \multicolumn{2}{|c|}{ Controls ( $n$ 217) } \\
\hline & Mean & SD & Mean & SD \\
\hline Age (years) & $44 \cdot 13$ & 1.73 & 43.39 & 2.57 \\
\hline BMI $\left(\mathrm{kg} / \mathrm{m}^{2}\right)$ & $30 \cdot 68^{*}$ & 2.79 & $21 \cdot 87$ & 1.58 \\
\hline SBP (mmHg) & $129 \cdot 09^{*}$ & $9 \cdot 23$ & $113 \cdot 34$ & $12 \cdot 74$ \\
\hline DBP (mmHg) & $82 \cdot 00^{*}$ & $6 \cdot 98$ & $74 \cdot 01$ & $8 \cdot 16$ \\
\hline $\mathrm{TC}(\mathrm{mmol} / \mathrm{l})$ & $5 \cdot 00^{*}$ & 0.51 & 4.54 & 0.77 \\
\hline TAG (mmol/l) & $1.41^{*}$ & 0.24 & $1 \cdot 10$ & 0.32 \\
\hline HDL-C (mmol/l) & $1.33^{\star}$ & 0.09 & 1.56 & 0.23 \\
\hline FBG (mmol/l) & 4.54 & 0.61 & 4.52 & 0.63 \\
\hline $2 \mathrm{~h}-\mathrm{PG}(\mathrm{mmol} / \mathrm{l})$ & $5 \cdot 76^{\star}$ & 1.12 & 5.47 & 1.07 \\
\hline CRP (ng/ml) & $2 \cdot 42^{\star}$ & 1.46 & 1.26 & 0.66 \\
\hline IL-6 (pg/ml) & $5 \cdot 57^{\star}$ & 3.93 & 4.58 & 2.99 \\
\hline SOD (U/ml) & $91 \cdot 29^{*}$ & 23.49 & $102 \cdot 61$ & $25 \cdot 2$ \\
\hline MDA (nmol/ml) & $2 \cdot 96^{*}$ & 0.98 & 2.51 & 0.89 \\
\hline GSH-PX ( $\mu \mathrm{mol} / \mathrm{l})$ & $158 \cdot 90^{\star}$ & 36.43 & $167 \cdot 39$ & 33.01 \\
\hline \multicolumn{5}{|c|}{ Amino acids (mmol/l) } \\
\hline His & $172 \cdot 47^{\star} \dagger$ & 41.71 & $202 \cdot 07$ & $54 \cdot 34$ \\
\hline Arg & $98.58^{*} \dagger$ & $21 \cdot 84$ & $108 \cdot 62$ & $22 \cdot 24$ \\
\hline Thr & $157 \cdot 69^{*} \dagger$ & $40 \cdot 07$ & $174 \cdot 16$ & 39.94 \\
\hline Gly & $113.42^{*} \dagger$ & $24 \cdot 23$ & $124 \cdot 25$ & $27 \cdot 69$ \\
\hline Lys & $69.91^{*} \dagger$ & $18 \cdot 10$ & 79.91 & $22 \cdot 30$ \\
\hline Ser & $84 \cdot 70^{*} \dagger$ & $26 \cdot 63$ & $93 \cdot 67$ & 24.53 \\
\hline Ile & $39 \cdot 75$ & $11 \cdot 16$ & $44 \cdot 32$ & $12 \cdot 04$ \\
\hline Pro & 108.42 & $30 \cdot 70$ & $103 \cdot 28$ & $22 \cdot 30$ \\
\hline Glu & $89 \cdot 71$ & 27.56 & 87.75 & 21.61 \\
\hline Met & $27 \cdot 85$ & $14 \cdot 65$ & $30 \cdot 51$ & $16 \cdot 63$ \\
\hline Leu & 85.49 & 31.89 & 87.47 & 28.72 \\
\hline Phe & $62 \cdot 84$ & $23 \cdot 11$ & 63.47 & 20.95 \\
\hline Val & $110 \cdot 21$ & $45 \cdot 65$ & 111.74 & 50.09 \\
\hline
\end{tabular}

SBP, systolic blood pressure, DBP, diastolic blood pressure; TC, total cholesterol; HDL-C, HDL-cholesterol; FBG, fasting blood glucose; $2 \mathrm{~h}-\mathrm{PG}, 2$-h postload blood glucose concentration; CRP, C-reactive protein; SOD, superoxide dismutase; MDA, malondialdehyde; GSH-PX, glutathione peroxidase.

${ }^{*}$ Mean values were significantly different from those of non-obese controls $(P<0.001$; Student's $t$ test).

† Mean values were significantly different $(P<0.001$; analysis made by ANCOVA), adjusted for age, smoking, alcohol use, menopause and physical activity at work and at leisure. 
for age, smoking, alcohol use, menopause, physical activity at work and at leisure, serum histidine, arginine, threonine, glycine, lysine and serine were found to be significantly lower in obese women in comparison to non-obese controls. The difference was the greatest for histidine.

\section{Multiple linear regression between serum amino acids and inflammatory and oxidative biomarkers}

As shown in Table 2, among the serum histidine, arginine, threonine, glycine, lysine and serine, both histidine and arginine were negatively associated with IL-6, CRP and MDA, and positively associated with SOD; histidine was positively associated with glutathione peroxidase in the overall subjects. The results were consistent as the confounders were included step by step in each model.

In addition, as shown in Table S1 (the supplementary material for this article can be found at http://www.journals. cambridge.org/bjn), both histidine and arginine were negatively associated with IL-6, CRP and MDA, and positively associated with SOD in obese women. Histidine was positively associated with SOD and negatively associated with MDA in non-obese controls.

\section{Discussion}

In the present study, we found that the serum amino acid profiles could separate between obese and non-obese women. Among the thirteen kinds of amino acids determined, six serum amino acids (histidine, arginine, threonine, glycine, lysine and serine) were significantly lower in obese women

Table 2. Multiple linear regression between serum amino acids and inflammatory and oxidative stress biomarkers in overall subjects

(Standardised coefficients, $\beta$ )†

\begin{tabular}{|c|c|c|c|c|c|}
\hline & CRP & IL-6 & SOD & MDA & GSH-PX \\
\hline \multicolumn{6}{|c|}{ Model 1} \\
\hline $\mathrm{His}$ & $-0.229^{\star \star}$ & $-0.139^{*}$ & $0.243^{\star \star}$ & $-0.144^{*}$ & $0.172^{*}$ \\
\hline Arg & $-0.171^{\star}$ & $-0.163^{*}$ & $0.141^{*}$ & $-0.115^{\star}$ & -0.066 \\
\hline \multicolumn{6}{|c|}{ Model 2} \\
\hline His & $-0.222^{\star \star}$ & $-0.140^{*}$ & $0.249^{\star \star}$ & $-0.139^{\star}$ & $0.176^{*}$ \\
\hline Arg & $-0.174^{\star}$ & $-0.163^{\star}$ & $0 \cdot 144^{*}$ & $-0.116^{\star}$ & -0.068 \\
\hline \multicolumn{6}{|c|}{ Model 3} \\
\hline His & $-0.226^{\star \star}$ & $-0.137^{\star}$ & $0.252^{\star \star}$ & $-0.137^{\star}$ & $0.176^{\star}$ \\
\hline Arg & $-0.173^{*}$ & $-0.162^{*}$ & $0 \cdot 144^{\star}$ & $-0.116^{\star}$ & -0.068 \\
\hline \multicolumn{6}{|c|}{ Model 4} \\
\hline $\mathrm{His}$ & $-0.227^{\star *}$ & $-0.135^{*}$ & $0.248^{\star *}$ & $-0.138^{\star}$ & $0.174^{\star}$ \\
\hline Arg & -0.176 & $-0.163^{*}$ & $0.139^{*}$ & $-0.125^{\star}$ & -0.071 \\
\hline \multicolumn{6}{|c|}{ Model 5} \\
\hline $\mathrm{His}$ & $-0.229^{\star \star}$ & $-0.141^{*}$ & $0.253^{\star \star}$ & $-0.138^{*}$ & $0 \cdot 171^{*}$ \\
\hline Arg & $-0.179^{\star}$ & $-0.158^{*}$ & $0.138^{*}$ & $-0.125^{\star}$ & -0.077 \\
\hline \multicolumn{6}{|c|}{ Model 6} \\
\hline His & $-0.171^{*}$ & $-0.128^{*}$ & $0.228^{\star \star}$ & $-0.112^{*}$ & $0.149^{*}$ \\
\hline Arg & $-0.177^{\star}$ & $-0 \cdot 160^{*}$ & $0 \cdot 142^{*}$ & $-0.122^{\star}$ & -0.080 \\
\hline
\end{tabular}

CRP, C-reactive protein; SOD, superoxide dismutase; MDA, malondialdehyde; $\mathrm{GSH}-\mathrm{PH}$, glutathione peroxidase.

Values were significantly different: ${ }^{\star} P<0.005,{ }^{\star \star} P<0.001$.

$\dagger$ Multiple linear regression: potential confounders including age, smoking, alcohol use, menopause and physical activity at work and at leisure, and BMI were included step by step in Models 1-6; threonine, glycine, lysine and serine were not shown in the table, for their standardised coefficients were not significant. than in the non-obese controls, although obese and nonobese subjects had similar dietary protein intake (Table 1).

The difference of serum histidine (mean histidine level in obese women was lower than in the controls by $14.5 \%$ ) was the greatest of the amino acids between the two groups, which indicated that histidine might be used as a biomarker of human obesity. Our study reported a lower serum histidine in obese women for the first time. However, subjects with some other conditions were also reported to have lower histidine concentrations. Watanabe et $a l^{(11)}$ reported that plasma histidine concentrations were significantly lower in chronic kidney disease patients with a history of CVD, proteinenergy wasting and inflammation. Wijekoon et al. ${ }^{(12)}$ found that fat Zucker diabetic rats had significantly lower plasma histidine than that of lean Zucker diabetic rats. Lee et al. ${ }^{(13)}$ showed that diabetic Balb/cA mice had lower plasma histidine compared with non-diabetic mice. It is obvious that subjects in the previous human and animal studies shared the same feature: inflammation and oxidative stress. According to our results and previous evidence, inflammation and oxidative stress in obese subjects might have a close relationship with a low histidine level.

Further, we found that histidine was significantly negatively associated with inflammation (IL-6: $\beta=-0 \cdot 210$ and CRP: $\beta=-0 \cdot 180, P<0.05$ ) and oxidative stress (SOD: $\beta=0.327$ and MDA: $\beta=-0.282, P<0.05)$ in obese and overall subjects (Table 2). Findings of previous studies might explain these relationships. First, inflammatory status might lead to the low histidine levels. Several studies have found that histidine supplementation could reduce the levels of IL- 6, TNF- $\alpha$ and CRP in animal models with liver injury or diabetes ${ }^{(13,14)}$. Son et al. ${ }^{(15)}$, in their in vitro study, reported that histidine inhibited the $\mathrm{H}_{2} \mathrm{O}_{2}$ - and TNF- $\alpha$-induced IL- 8 secretion at the transcriptional level in intestinal epithelial cells. Second, because histidine was a free-radicals scavenger and could chelate divalent metal ions in animal and in vitro studies ${ }^{(16,17)}$, oxidative stress in obese subjects might be another reason for this negative relationship. However, it was still unclear regarding the causation of lower histidine level in obese women. Further animal studies should be designed to determine if patientsinduced obesity could lead to the low histidine level.

Arginine has been well explored in its nutritional and therapeutic roles in treating and preventing human and animal metabolic disorders. Its low level in obese subjects might relate to its bio-functions. Arginine is the substrate for the synthesis of NO and has numerous useful physiological properties and immunomodulatory effects. In diabetic animal models, arginine supplementation was found to reduce white fat mass and endothelium-dependent relaxation, and also to increase the brown adipose tissue ${ }^{(18,19)}$. One clinical trial investigated the supplementation of arginine for $21 \mathrm{~d}$ in human subjects and found that arginine increased the antioxidant capacity in the obese humans ${ }^{(20)}$. Arginine treatment was reported to have decreased inflammatory cytokine levels in chronic renal failure and diabetic rats ${ }^{(21,22)}$.

There were six kinds of amino acids found lower in obese subjects with a large sample size. Among them, histidine, threonine and lysine are essential amino acids; arginine, 
glycine and serine are non-essential amino acids. There could be a higher level of catabolism or utilisation for the essential amino acids; and higher level of utilisation or lower for de novo synthesis of non-essential amino acids in obese subjects. However, as to why the lower circulating levels of these amino acids were found in obese subjects, was still unclear. Whether there are any functional interactions with these amino acids still needs more investigation. As we all know, amino acids were not only the common nutrients, but also cell signalling molecules, regulators of gene expression and the protein phosphorylation cascade $^{(24)}$. Our findings suggest that more attention should be paid to the imbalanced amino acid metabolism in obese subjects.

In the present study, we found that obese subjects had the most significant histidine level and that histidine was negatively associated with inflammation and oxidative stress in obese women. In addition, there have been reports indicating that histidine could suppress appetite and influence body weight through its conversion into neuronal histamine in the hypothalamus of female subjects ${ }^{(25,26)}$. Arginine also has the effects of anti-inflammation, anti-oxidative stress and regulating of the vascular functions. Thus, it is interesting to uncover if obese subjects could benefit from histidine or arginine supplementation in their daily diet.

There are still some limitations in the present study. First, this study only included middle-aged female subjects as they have higher obesity prevalence. The findings from the sample of females alone in this case-control study may not be generalised to the total population. Second, this case-control study may not provide fully convincing evidence of the causal effects of histidine and arginine on chronic inflammation and oxidative stress in obese human subjects; hence, further animal and clinical studies are necessary to clarify these associations

In conclusion, our study indicated that amino acid metabolic disorder might exist in obese women. Overall, six kinds of serum amino acids were significantly lower in obese women; serum amino acid profiles could discriminate between obese and non-obese women; and histidine and arginine have close relationships with inflammation and oxidative stress in obese women.

\section{Acknowledgements}

The authors appreciate the efforts and collaboration of colleagues from the Harbin Center for Disease Control and Prevention in the present study. This study was supported by the National Natural Science Fund of China (nos. 81172649, 81102112 and 81130049). The contributions of the authors to this study are as follows: Y. L. and C.-H. S. were responsible for the study concept and design; R.-N. F., Y.-C. $\mathrm{N}$. and Z. K. performed the experiment and collection of data; K. L. and J. W. carried out the analysis and interpretation of the data; R.-N. F., Y.-C. N. and Y. H. were responsible for the drafting of the manuscript; Y. L. and C.-H. S. contributed also to the critical revision of the manuscript for its intellectual content; K. L. and Y. H. performed the statistical analysis; and Y. L. and C.-H. S. were responsible for the obtaining of funding and supervision. None of the authors had a conflict of interest.

\section{References}

1. Wolongevicz DM, Zhu L, Pencina MJ, et al. (2010) Diet quality and obesity in women: the Framingham nutrition studies. Br J Nutr 103, 1223-1229.

2. Trayhurn P, Wang B \& Wood IS (2008) Hypoxia in adipose tissue: a basis for the dysregulation of tissue function in obesity? Br J Nutr 100, 227-235.

3. Franssen R, Monajemi H, Stroes ES, et al. (2008) Obesity and dyslipidemia. Endocrinol Metab Clin North Am 37, 623-633, viii.

4. Becker S, Dossus L \& Kaaks R (2009) Obesity related hyperinsulinaemia and hyperglycaemia and cancer development. Arch Physiol Biochem 115, 86-96.

5. Aikawa M, Sugiyama S, Hill CC, et al. (2002) Lipid lowering reduces oxidative stress and endothelial cell activation in rabbit atheroma. Circulation 106, 1390-1396.

6. Lamb RE \& Goldstein BJ (2008) Modulating an oxidativeinflammatory cascade: potential new treatment strategy for improving glucose metabolism, insulin resistance, and vascular function. Int J Clin Pract 62, 1087-1095.

7. Aviram M (2009) Hyperlipidaemia and cardiovascular disease: inflammation and oxidative stress in diabetic patients. Curr Opin Lipidol 20, 258-259.

8. Rytter E, Vessby B, Asgard R, et al. (2009) Glycaemic status in relation to oxidative stress and inflammation in well-controlled type 2 diabetes subjects. Br J Nutr 101, 1423-1426.

9. Zhou BF (2002) Predictive values of body mass index and waist circumference for risk factors of certain related diseases in Chinese adults - study on optimal cut-off points of body mass index and waist circumference in Chinese adults. Biomed Environ Sci 15, 83-96.

10. Li Y, Wang C, Zhu K, et al. (2010) Effects of multivitamin and mineral supplementation on adiposity, energy expenditure and lipid profiles in obese Chinese women. Int $J$ Obes (Lond) 34, 1070-1077.

11. Watanabe M, Suliman ME, Qureshi AR, et al. (2008) Consequences of low plasma histidine in chronic kidney disease patients: associations with inflammation, oxidative stress, and mortality. Am J Clin Nutr 87, 1860-1866.

12. Wijekoon EP, Skinner C, Brosnan ME, et al. (2004) Amino acid metabolism in the Zucker diabetic fatty rat: effects of insulin resistance and of type 2 diabetes. Can J Physiol Pharmacol 82, 506-514.

13. Lee YT, Hsu CC, Lin MH, et al. (2005) Histidine and carnosine delay diabetic deterioration in mice and protect human low density lipoprotein against oxidation and glycation. Eur J Pharmacol 513, 145-150.

14. Yan SL, Wu ST, Yin MC, et al. (2009) Protective effects from carnosine and histidine on acetaminophen-induced liver injury. J Food Sci 74, H259-H265.

15. Son DO, Satsu H \& Shimizu M (2005) Histidine inhibits oxidative stress- and TNF-alpha-induced interleukin-8 secretion in intestinal epithelial cells. FEBS Lett 579, 4671-4677.

16. Babizhayev MA, Seguin MC, Gueyne J, et al. (1994) L-Carnosine (beta-alanyl-L-histidine) and carcinine (beta-alanylhistamine) act as natural antioxidants with hydroxylradical-scavenging and lipid-peroxidase activities. Biochem J 304, 509-516.

17. Lee JW, Miyawaki H, Bobst EV, et al. (1999) Improved functional recovery of ischemic rat hearts due to singlet oxygen 
scavengers histidine and carnosine. J Mol Cell Cardiol 31, $113-121$.

18. Fu WJ, Haynes TE, Kohli R, et al. (2005) Dietary L-arginine supplementation reduces fat mass in Zucker diabetic fatty rats. J Nutr 135, 714-721.

19. Wu G, Collins JK, Perkins-Veazie P, et al. (2007) Dietary supplementation with watermelon pomace juice enhances arginine availability and ameliorates the metabolic syndrome in Zucker diabetic fatty rats. $J$ Nutr $\mathbf{1 3 7}, 2680-2685$.

20. Lucotti P, Setola E, Monti LD, et al. (2006) Beneficial effects of a long-term oral L-arginine treatment added to a hypocaloric diet and exercise training program in obese, insulinresistant type 2 diabetic patients. Am J Physiol Endocrinol Metab 291, E906-E912.

21. Korish AA (2010) Multiple antioxidants and L-arginine modulate inflammation and dyslipidemia in chronic renal failure rats. Ren Fail 32, 203-213.
22. Pai $\mathrm{MH}$, Huang $\mathrm{KH}$, Wu $\mathrm{CH}$, et al. (2010) Effects of dietary arginine on inflammatory mediator and receptor of advanced glycation endproducts (RAGE) expression in rats with streptozotocin-induced type 2 diabetes. Br J Nutr 104, 686-692.

23. Huang CC, Tsai SC \& Lin WT (2008) Potential ergogenic effects of L-arginine against oxidative and inflammatory stress induced by acute exercise in aging rats. Exp Gerontol 43, 571-577.

24. Wu G (2009) Amino acids: metabolism, functions, and nutrition. Amino Acids 37, 1-17.

25. Yoshimatsu H, Chiba S, Tajima D, et al. (2002) Histidine suppresses food intake through its conversion into neuronal histamine. Exp Biol Med (Maywood) 227, 63-68.

26. Kasaoka S, Tsuboyama-Kasaoka N, Kawahara Y, et al. (2004) Histidine supplementation suppresses food intake and fat accumulation in rats. Nutrition 20, 991-996. 